\title{
Multi-Sensor Ensemble Classifier for Activity Recognition
}

\author{
Lingfei Mo ${ }^{1}$, Shaopeng Liu' ${ }^{2}$, Robert X. Gao ${ }^{2 *}$, Patty S. Freedson ${ }^{3}$
}

${ }^{1}$ School of Instrument Science and Engineering, Southeast University, Nanjing, China; ${ }^{2}$ Department of Mechanical Engineering, University of Connecticut, Storrs, CT, USA; ${ }^{3}$ Department of Kinesiology, University of Massachusetts, Amherst, MA, USA.

Email: rgao@engr.uconn.edu

Received 2012.

\begin{abstract}
This paper presents a multi-sensor ensemble classifier (MSEC) for physical activity (PA) pattern recognition of human subjects. The MSEC, developed for a wearable multi-sensor integrate measurement system (IMS),combines multiple classifiers based on different sensor feature sets to improve the accuracy of PA type identification.Experimental evaluation of 56 subjects has shown that the MSECis more effectivein assessing activities of varying intensitiesthan the traditional homogeneous classifiers. It is able to correctly recognize 6 PA types with an accuracy of $93.50 \%$, which is $7 \%$ higher than the non-ensemble support vector machine method. Furthermore, the MSECis effective in reducing the subject-to-subject variabilityin activity recognition.
\end{abstract}

Keywords:Physical Activity Assessment;Multi-Sensor Ensemble;Support Vector Machine.

\section{Introduction}

Physical activity (PA), defined as bodily movement generated by skeletal muscles [1]such as walking, joggingor sport activities, is important for maintaining health and preventing cardiovascular diseases, diabetes, and obesity. Accurate monitoring and assessment of PA under freeliving conditions provides information on the type and intensity of activities that the person has been engaged in, thus is of significant interest to the research community [1] and commercial companies.

The goal of PA assessment is to recognize the type, duration, and intensity of a broad range of physical activities and quantify the energy expenditureof the test subjectduring his/her daily life, as illustrated in Figure 1. In recent years, multi-sensor systems have been increasingly investigated for PA assessment. For example, multiple accelerometers have been placed at different locations on the bodyof test subjects [3]or combined with other types of sensors, such as respiratory sensor or GPS [4] for PA measurement. Combining advanced computational techniques such as machine learning and sensor fusion $[3,4]$, differentiation of various activities has shown to be improved. For example, a multi-sensor integrate measurement system (IMS) was developed with two accelerometers and one ventilation sensor to measure and assess the physical activity [5].

Recently, ensemble learning has been increasingly investtigated for pattern recognition [6]. An ensemble learning method combines multiple individual classifiers to obtain better predictive performance than that obtained by any of the constituent classifiers [7]. This is a technique that usually combines a number of weak classifiers together to produce a strong classifiers. Through combination of decisions from multiple classifiers or multiple sensors, recognition accuracy has shown to be improved effecttively [6]. For example, Ravi et al. combined multiple different classifiers to identify eight common activities of two subjects with a single tri-axial accelerometer [8]. These classifiers used the same four-feature (Mean, Standard Deviation, Energy and Correlation) datasets, and had obtained good recognition accuracies, especially by means ofMajority Voting. Lester et al. used AdaBoost [9] to select features and combined multiple weak classifiers, each of which accepting only a single feature as

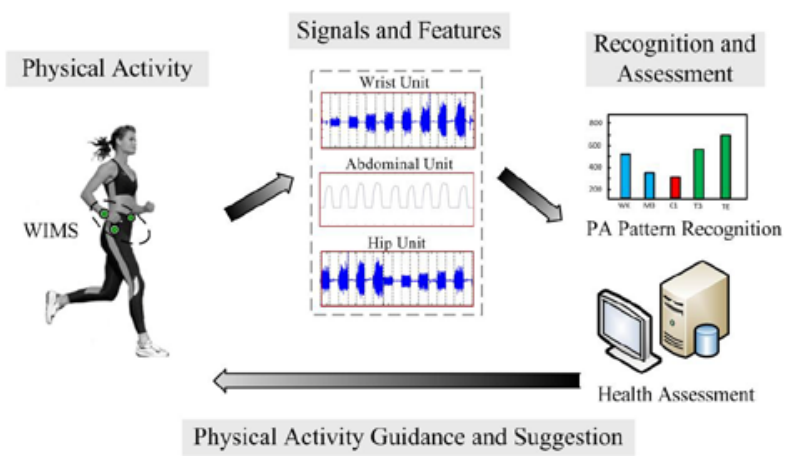

Figure 1. Physical activity assessment. 
input, and obtained good classification result from a weighted combination of the weak classifiers[10]. The eight sensors,inlcuding accelerometer, audio sensor, IR/ visible light, high frequency light, barometric pressure, humidity, temperature and compass, were integrated in a unit attached on the shoulder of the test subject, and tested by two subjects. For multi-sensor measurement system, combining different sensors at different body locations would reveal different characteristics of body movement and have different statistical distribution. Therefore, combining different classifiers based on the datasets of different sensors would have better identification results than a homogeneous classifier.

In this paper, a multi-sensor ensemble classifier (MSEC) for PA type identification is presentedfor a multi-sensor integrated measurement system (IMS) [5]. It combines multiple classifiers of SVM, based on different sensor datasets of the IMS. Due to different PA type identification accuracies of the different classifiers, an instance specific weight majority voting is proposed for the classifier combination. The performance of the MSECis experimentally evaluated by 56 human subjects performing free-living activities.

\section{Ensemble Learning}

\subsection{System Design}

The architecture of the multi-sensor ensemble classifieris shown in Figure 2. The sensor sets for ensemble are generated by choosing different sensors of the multi-sensor measurement system. Features corresponding to these sensor datasets are then extracted and selected. Multiple classifiers with different feature selection can be derived from each sensor set, and the diversity of the classifiers can be achieved by choosing the sensor set and feature combinations. For each classifier, a learning model is first selected and trained, with a part of the sensor data as testing dataset to evaluate the classifier. Each classifier has a decision result, and the final decision is thus obtained by combining the classification results from all the classifiers by the weight majority voting.

\subsection{Individual Classifiers}

Seven classifiers with different sensor datasets were devised for the ensemble system based on the three sensors in the IMS. Each sensor dataset consists of a cluster of classifiers, including 1) C1 (classifier cluster from the wrist accelerometer dataset), 2) C2 (classifier cluster from the hip accelerometer dataset), 3) C3 (classifier cluster from the abdominal ventilation sensor dataset), 4) C4 (classifier cluster from the hip accelerometer and the wrist accelerometer datasets), 5) C5 (classifier cluster from the hip accelerometer and the abdominal ventilation sensor datasets), 6) C6 (classifier cluster from the wrist accelerometer and the abdominal ventilation sensor datasets), and 7) C7 (classifier cluster from the hip and wrist accelerometer and the abdominal ventilation sensor datasets). Each classifier cluster consists of multiple (n) classifiers by different feature selection (random selection). As a result, a total of $7 \times \mathrm{n}$ classifiers and $7 \times \mathrm{n}$ testing results can be obtained. The final ensemble decision is obtained based on these $7 \times \mathrm{n}$ classifiers by instance specific weighted majority voting.

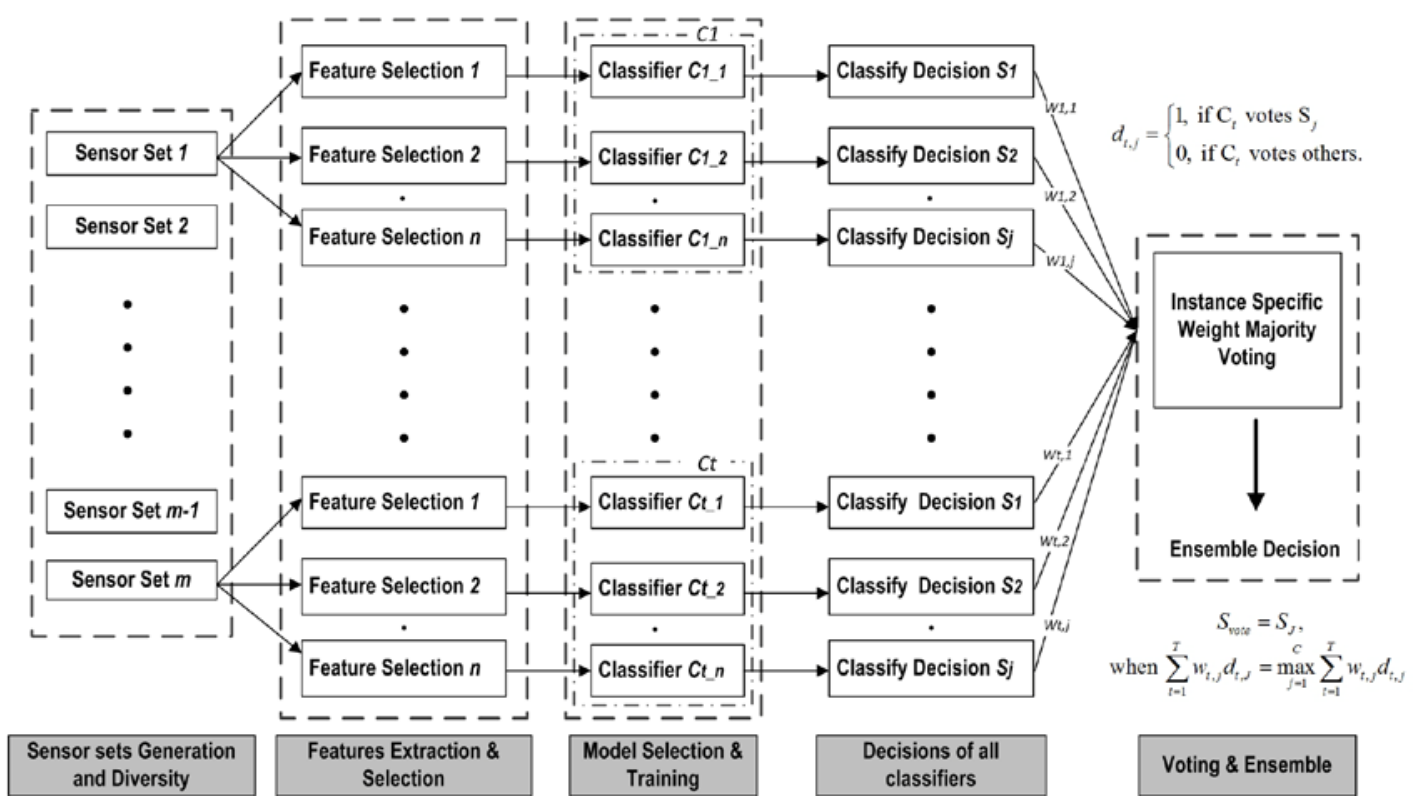

Figure 2. Multi-sensor ensemble system block diagram. 


\subsection{Activity Recognition}

For each single sensor, 7 time-domain features, namely the 10th, 25th, 50th (median), 75th, and 90th percentiles, the mean value, and the standard deviation, were extracted. In addition, a correlation feature between the hip accelerometer and the wrist accelerometer was also extracted, providing a measure for the coordination or variation between the upper limb and the body during an activity. For each accelerometer, two frequency domain features, energy and entropy were extracted. For the ventilation sensor, the dominant frequency of the respiratory signal obtained from a spectral analysis was extracted as the breathing frequency. These features were computed for every 30-second data segment, and linear scaling was then applied to the extracted features in the range of [0, $1]$, to avoid that features of greater numeric values would overwhelm those in the smaller numeric ranges.As a result, a total of 63 features (50 time-domain and 13 frequency-domain features) were extracted. To achieve the diversity of the input of each classifier, $70 \%$ of the features were selected randomly from the overall feature sets for training classifiers.

The SVM algorithm was chosen as the base classifier of the ensemble system, and the selected features were used as inputs to the SVM classifier. A two-step procedure was taken for predicting the types of physical activity. First, a training data set that consists of the selected features from all the 56 subjects but one was constructed for building the SVM model and selecting the penalty parameter and Gaussian kernel parameter. The model parameters were selected through a "grid-search" with 5-fold cross validation. The parameters that yielded the highest recognition rate were chosen during the process. Second, upon completion of the training, the SVM model was applied to the feature set of the subject that was left out in the training process, to predict the activity type reflected in the 30-second data segments. Such a twostep procedure constitutes a "leave-one-subject-out" cross validation, and was executed on each subject data.

\section{Experimental Evaluation}

\subsection{Design of Experiments}

A total of 56 subjects (26 male and 30 female) were recruited forphysical activity assessment, with the following characteristics, expressed in terms of the mean \pm standard deviation:

1) age $=38.7 \pm 11.6$ years,

2) mass $=71.1 \pm 14.5 \mathrm{~kg}$,

3) height $=169.3 \pm 9.1 \mathrm{~cm}$ and

4) body mass index $=24.7 \pm 4.2 \mathrm{~kg} / \mathrm{m}^{2}$.

Each subject performed 6 types of activities of varying intensities, which are commonly seen in daily lives as illustratedin Table 1. For each subject, the actual PA types and times performed by the subjects were recorded, and sensor data whendifferent PA types were performed were collected by the IMS (as shown in Figure 2) correspondingly [7]. Each PA type was performed for 7 minutes, followed by a 2-minute rest period.

\subsection{Individual Classifier Results}

In order to ensemble different classifiers by the instance specific weight majority voting method,it is necessary to first investigatethe accuracies of the different classifier clusters. Furthermore, since these classifiers use features from different sensor datasets, they yield different accuracies and confidences on identifying the PA types. The average accuracies of the classifiers on the different PA types are first calculated, as shown in Table 2 . It is seen that these classifiers have yielded different accuracies on PA type identifications, which is due to the fact that different sensor combinations are sensitive to different PA types.

\subsection{Ensemble Results}

The performance of the ensemble system is based on the number and accuracies of the classifiers integrated for the ensemble learning. Various ensemble classifiers had been evaluated. The definitions of the different ensemble classifiers are: E1-E7 integrate the classifiers within each sensor cluster C1-C7, respectively, E8 integrates classifiers of C1, C2 and C3, E9 integrates classifiers of C4, C5, and C6, and E10 integrates classifiers C1-C7.

Table 1. Physical Activities types for testing.

\begin{tabular}{lll}
\hline Activities & Category & Abbr. \\
\hline Computer work & Sedentary activity & $C W$ \\
Moving boxes & & $M B$ \\
Cycling with 1-kp resistance & Household and other & $C 1$ \\
Treadmill at 3.0 mph & Moderate locomotion & T3 \\
Treadmill at 4.0 mph 5\% grade & Vigorous activity & T4-5 \\
Tennis & & $T E$ \\
\hline
\end{tabular}

Table 2. Classification accuracies of different sensor classifiers on different PA types

\begin{tabular}{|c|c|c|c|c|c|c|c|}
\hline & & \multicolumn{6}{|c|}{ PA Types } \\
\hline & & $C W$ & $M B$ & C1 & T3 & $T 4-5$ & $T E$ \\
\hline \multirow{7}{*}{ 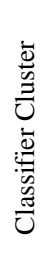 } & C1 & $89.5 \%$ & $94.7 \%$ & $81.6 \%$ & $80.1 \%$ & $61.7 \%$ & $72.1 \%$ \\
\hline & $C 2$ & $88.2 \%$ & $71.7 \%$ & $86.2 \%$ & $91.7 \%$ & $86.0 \%$ & $71.5 \%$ \\
\hline & C3 & $46.8 \%$ & $54.5 \%$ & $37.7 \%$ & $47.2 \%$ & $37.9 \%$ & $25.6 \%$ \\
\hline & C4 & $91.2 \%$ & $87.1 \%$ & $89.5 \%$ & $91.4 \%$ & $86.2 \%$ & $73.4 \%$ \\
\hline & C5 & $83.8 \%$ & $73.0 \%$ & $87.4 \%$ & $91.7 \%$ & $85.4 \%$ & $70.9 \%$ \\
\hline & C6 & $91.2 \%$ & $76.3 \%$ & $81.3 \%$ & $82.7 \%$ & $66.5 \%$ & $96.7 \%$ \\
\hline & C7 & $92.9 \%$ & $72.9 \%$ & $91.0 \%$ & $94.0 \%$ & $92.4 \%$ & $85.4 \%$ \\
\hline
\end{tabular}




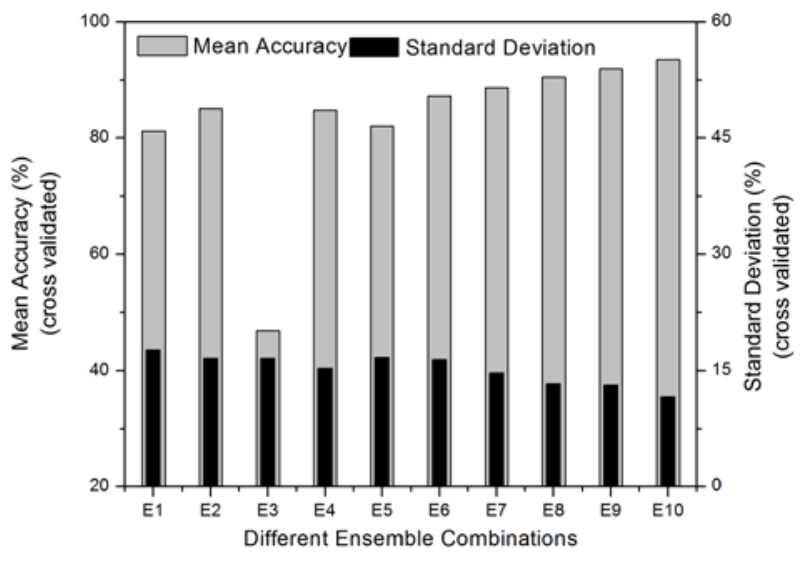

Figure 3. Ensemble classifier result comparison.

Figure 3 illustrates the PA classification results of the various ensemble classifiers E1-E10. It is seen that in general, the more classifiers and sensor datasets are included in the ensemble classifier, the better the result has been. For example, classifier E10 integrates all the classifiers (total 21), and hasyielded the best classification mean accuracy of $93.5 \%$ with the smallest standard deviation of $11.6 \%$.

\section{Conclusions}

In this study, a multi-sensor ensemble classifier is designed for physical activity recognition, as a critical component of a multi-sensor integrated measurement system. Specifically, MSEC integrates data measured by three sensors, and fuses the multiple classifiers by performing instance specific weight majority voting. Compared with non-ensemble classifier, the MSECmethod has shown higher mean accuracies and lower standard deviations, thus demonstrateing better generalization capability.

Although promising, the MSEC is generally computationally intensive, requiring more computational resources than non-ensemble single classifier to achieve good performance. Furthermore, there are still questions that remained unanswered, e.g., 1) how many sensors and what type of sensors (including the locations where the sensors are attached) are required for PA assessment, and 2) what type of features and classifiers are suitable and optimal for the ensemble system. Research is being continued to systematically investigate the developed MSEC algorithm in terms of effectiveness and computational efficiency for improved PA classification.

\section{Acknowledgement}

The authors gratefully acknowledge funding provided for this research by the National Institutes of Health under Grant UO1 A130783.

\section{REFERENCES}

[1] C. Caspersen, K. Powell, and G. Christenson, "Physical activity, exercise, and physical fitness: definitions and distinctions for health-related research", Public health reports, Vol. 100, No. 2, pp. 126-131, 1985.

[2] D. Hendelman, K. Miller, C. Bagget, E. Debold, and P. Freedson, "Validity of accelerometry for the assessment of moderate intensity physical activity in the field", Medicine and Science in Sports and Exercise, Vol. 32, No. 9, pp. 442-449, 2000.

[3] L. Bao, and S. Intille, "Activity recognition from user-annotated acceleration data”, Pervasive Computing, Vol. 3001, pp. 1-17, 2004.

[4] M. Ermes, J. Parkka, J. Mantyjarvi, and I. Korhonen, "Detection of daily activities and sports with wearable sensors in controlled and uncontrolled conditions", IEEE Transactions on Information Technology in Biomedicine, Vol. 12, No. 1, pp. 20-26, 2008.

[5] S. Liu, R. Gao, and P. Freedson, "Design of a wearable multi-sensor system for physical activity assessment”, Proc. IEEE/ASME Int. Conf. on Advanced Intelligent Mechatronics, pp. 254-259, 2010.

[6] J. Lester, T. Choudhury, and G. Borriello, "A practical approach to recognizing physical activities”, Pervasive Computing, pp. 1-16, 2006.

[7] L. Breiman, "Bagging predictors", Machine learning, Vol.24, No. 2, pp. 123-140, 1996.

[8] N. Ravi, N. Dandekar, P. Mysore, and M. Littman, “Activity recognition from accelerometer data”, Proceedings of the Seventeenth Conference on Innovative Applications of Artificial Intelligence(IAAI 2005), pp. 1541-1546, 2005.

[9] M. Gashler, C. Giraud-Carrier, and T. Martinez, "Decision tree ensemble: small heterogeneous is better than large homogeneous", IEEE Seventh International Conference on Machine Learning and Applications, San Diego, CA, USA, pp. 900-905, 2008.

[10] D. Opitz, and R. Maclin, "Popular ensemble methods: An empirical study”, Journal of Artificial Intelligence Research, Vol. 11, No. 1, pp. 169-198, 1999. 